\title{
One-Pot Procedure for Diazo Transfer and Azide-Alkyne Cycloaddition: Triazole Linkages from Amines
}

\author{
Henning S. G. Beckmann and Valentin Wittmann* \\ Fachbereich Chemie, Universität Konstanz, D-78457 Konstanz, Germany
}

\section{SUPPORTING INFORMATION}

\section{General Methods}

Syntheses under microwave irradiation were performed in sealed tubes using a Biotage $A B$ SmithSynthesizer. TLC was performed on Merck Silica Gel $60 \mathrm{~F}_{254}$ aluminum sheets. Reagents used for developing plates include Cer-Reagent $(5 \mathrm{~g}$ molybdatophosphoric acid, $2.5 \mathrm{~g}$ ceric sulphate tetrahydrate, $25 \mathrm{ml}$ sulfuric acid and $225 \mathrm{ml}$ water), ethanolic ninhydrine (3\% w/v), ethanolic sulfuric acid (15\% v/v) and detection by UV light was used when applicable. Flash column chromatography was performed on Macherey-Nagel Silica Gel 60 (0.04-0.063 mm; $230-400$ mesh ASTM). ${ }^{1} \mathrm{H}$ and ${ }^{13} \mathrm{C}$ NMR spectra were recorded at room temperature on a Bruker Avance DRX 600 instrument. ${ }^{1} \mathrm{H}$ chemical shifts are referenced to residual protic solvent $\left(\mathrm{CDCl}_{3}, \delta_{\mathrm{H}}=7.26 \mathrm{ppm}\right.$; DMSO- $\mathrm{d}_{6}, \delta_{\mathrm{H}}=$ $2.50 \mathrm{ppm}) .{ }^{13} \mathrm{C}$ chemical shifts are referenced to the solvent signal $\left(\mathrm{CDCl}_{3}, \delta_{\mathrm{C}}=77.0 \mathrm{ppm} ; \mathrm{DMSO}-\mathrm{d}_{6}\right.$, $\delta_{\mathrm{C}}=39.4 \mathrm{ppm}$ ). ESI mass spectra were recorded on a Bruker Daltonics Esquire 3000 plus instrument. Combustion elemental analyses were performed on an elementar vario EL analyzer.

Warning: In the case of reactions with azide sources in dichloromethane, formation of explosive diazidomethane has been reported. ${ }^{1}$ Therefore, the triflyl azide solution in dichloromethane should always be prepared freshly and the reaction time of $2 \mathrm{~h}$ should not be exceeded. Special caution should also be exercised during the workup procedure (especially during evaporation of the reaction mixture) due to excess triflyl azide and possibly formed copper azides, although we never observed any incident.

\section{General Procedure 1: Sequential One-Pot Process for Diazo Transfer and Azide-Alkyne Cycloaddition Using $\mathrm{CuSO}_{4}$ and Sodium Ascorbate}

Triflyl azide ( $\mathrm{TfN}_{3}$ ) was freshly prepared prior to each reaction. ${ }^{2} \mathrm{NaN}_{3}$ (6 eq per substrate amine) was dissolved in a minimum volume of water (solubility of $\mathrm{NaN}_{3}$ in water is approximately $0.4 \mathrm{~g} / \mathrm{ml}$ ). At $0{ }^{\circ} \mathrm{C}$ an equal volume of dichloromethane was added and triflic anhydride $\left(\mathrm{Tf}_{2} \mathrm{O}\right)$ (3 eq) was added dropwise to the vigorously stirred solution. After stirring for $2 \mathrm{~h}$ at $0{ }^{\circ} \mathrm{C}$ the aqueous phase was once 
extracted with the same volume of dichloromethane. The combined organic phases were washed with sat. $\mathrm{NaHCO}_{3}$ solution and used without further purification.

The substrate amine, $\mathrm{CuSO}_{4}(2 \mathrm{~mol} \%)$ and $\mathrm{NaHCO}_{3}(1 \mathrm{eq})$ were dissolved/suspended in the same volume of water as the volume of the $\mathrm{TfN}_{3}$ solution to be employed. The $\mathrm{TfN}_{3}$ solution was added, followed by addition of methanol until the solution became homogeneous. The reaction was stirred at room temperature (ca. $30 \mathrm{~min}$ ) until TLC showed complete conversion of the starting amine.

Then the acetylene component ( 1 or $1.1 \mathrm{eq})$, TBTA ( $5 \mathrm{~mol} \%)$ and sodium ascorbate $(10 \mathrm{~mol} \%$ per substrate amine) were added and the reaction was heated to $80^{\circ} \mathrm{C}$ in the microwave oven until TLC showed complete conversion of the azide intermediate (10 - $30 \mathrm{~min})$.

The reaction mixture was diluted with water and the organic solvents were removed under reduced pressure. If the product precipitates, it was collected by filtration. If not, the water was removed by lyophilization. (In order to remove copper salts, it is advisable to stir the solution with CupriSorb resin and filter it before lyophilization.) The crude product was purified by flash chromatography.

\section{1-Benzyl-4-phenyl-1H-1,2,3-triazole (3)}

\section{Method 1: According to General Procedure 1}

Benzylamine 1 (54 mg, $0.50 \mathrm{mmol})$ and phenylacetylene $2(55 \mu \mathrm{l}, 0.50 \mathrm{mmol})$ were reacted according to general procedure 1. After flash chromatography (petroleum ether / ethyl acetate 2:1) 3 was isolated as a white powder (105 mg, $94 \%)$. The ${ }^{1} \mathrm{H}$ and ${ }^{13} \mathrm{C}$ NMR spectroscopic data were in agreement with those published. ${ }^{3}$

Elemental Analysis: calcd. C $76.57 \%$, H $5.57 \%$, N $17.86 \%$; found: C $76.28 \%$, H $5.45 \%$, N $17.83 \%$.

\section{Method 2: According to General Procedure 1 Using Toluene and Isopropyl Alcohol}

The procedure is the same as described above except that toluene is used instead of dichloromethane and isopropyl alcohol instead of methanol. In this case 20 mol\% sodium ascorbate was used.

\section{Method 3: Sequential One-Pot Process for Diazo Transfer and Azide-Alkyne Cycloaddition Using} $\mathrm{CuSO}_{4}$ and $\mathrm{Cu}$ Powder

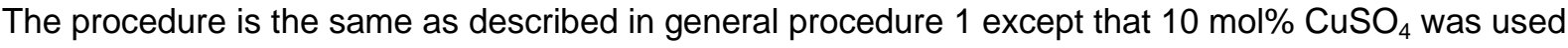
instead of $2 \mathrm{~mol} \%$ and Cu powder (30 mol\%) instead of sodium ascorbate.

Method 4: One-Step One-Pot Process for Diazo Transfer and Azide-Alkyne Cycloaddition Using Sodium Ascorbate or Cu Powder as Reducing Agent

$\mathrm{TfN}_{3}$ was prepared as described above. The substrate amine, the acetylene component (1 eq) and $\mathrm{NaHCO}_{3}(1 \mathrm{eq})$ were suspended in the same volume of water as the volume of the $\mathrm{TfN}_{3}$ solution to be 
employed. Then either 2 mol\% $\mathrm{CuSO}_{4}, 5$ mol\% TBTA and 10 mol\% sodium ascorbate per substrate amine or $10 \mathrm{~mol} \% \mathrm{CuSO}_{4}$ and $30 \mathrm{~mol} \% \mathrm{Cu}$ powder per substrate amine were added. The $\mathrm{TfN}_{3}$ solution was added, followed by addition of methanol until the solution became homogeneous. The reaction was heated to $80^{\circ} \mathrm{C}$ in the microwave oven until TLC showed complete conversion of the starting material. The workup procedure is the same as described in general procedure 1.

\section{1-(p-Methoxybenzyl)-4-phenyl-1H-1,2,3-triazole (9)}

p-Methoxybenzylamine 4 (69 $\mathrm{mg}, 0.50 \mathrm{mmol})$ and phenylacetylene $2(55 \mu \mathrm{l}, 0.50 \mathrm{mmol}$ ) were reacted according to general procedure 1 at $120{ }^{\circ} \mathrm{C}$ in the microwave oven. After flash chromatography (petroleum ether / ethyl acetate 2:1) 9 was isolated as a white powder $(104 \mathrm{mg}, 78 \%)$. The ${ }^{1} \mathrm{H}$ and ${ }^{13} \mathrm{C}$ NMR spectroscopic data were in agreement with those published. ${ }^{4}$

Elemental Analysis: calcd. C $72.43 \%$, H $5.70 \%$, N $15.84 \%$; found: C $72.43 \%$, H $6.08 \%$, N $16.13 \%$.

\section{Ethyl-2-(4-phenyl-1H-1,2,3-triazol-1-yl) acetate (10)}

Glycine ethyl ester hydrochloride $5(70 \mathrm{mg}, 0.50 \mathrm{mmol})$ and phenylacetylene $2(55 \mu \mathrm{l}, 0.50 \mathrm{mmol})$ were reacted according to general procedure 1 using 2 eq of $\mathrm{NaHCO}_{3}$. After flash chromatography (petroleum ether / ethyl acetate 2:1) 10 was isolated as a white powder (94 mg, $81 \%$ ). The ${ }^{1} \mathrm{H}$ and ${ }^{13} \mathrm{C}$ NMR spectroscopic data were in agreement with those published. ${ }^{5}$

Elemental Analysis: calcd. C $62.33 \%$, H $5.67 \%$, N $18.17 \%$; found: C $61.99 \%$, H $5.75 \%$, N $18.19 \%$.

\section{(2S)-2-(9'-Fluorenylmethyloxycarbonylamino)-6-(4-phenyl-1H-1,2,3-triazol-1-yl)hexanoic acid}

(11)

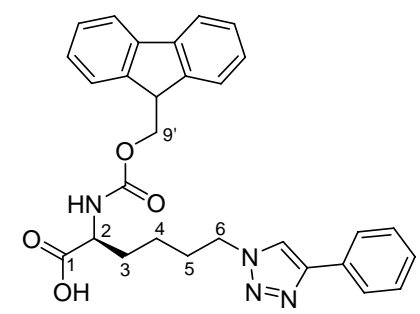

Fmoc-Lys-OH 6 (81 mg, $0.22 \mathrm{mmol}$ ) and phenylacetylene 2 (24 $\mu \mathrm{l}, 0.22 \mathrm{mmol}$ ) were reacted according to general procedure 1 using $30 \mathrm{~mol} \% \mathrm{Na}$ ascorbate. After flash chromatography $\left(\mathrm{CHCl}_{3} / \mathrm{MeOH} / \mathrm{AcOH}\right.$ 96:3:1) 11 (103 mg, $94 \%$ ) was isolated as a white powder.

${ }^{1} \mathrm{H}$ NMR $\left(\mathrm{CDCl}_{3}, 600 \mathrm{MHz}\right): \delta=7.77-7.71(\mathrm{~m}, 5 \mathrm{H} ; \mathrm{Ar}-\mathrm{H}$ and triazole-5), $7.58-7.53(\mathrm{~m}, 2 \mathrm{H} ; \mathrm{Ar}-\mathrm{H})$, 7.40 - 7.34 (m, 4 H; Ar-H), 7.32 - 7.29 (m, 1 H; Ar-H), 7.29 - 7.24 (m, 2 H; Ar-H), 5.63 - 5.58 (br. d, 1 $\mathrm{H} ; \mathrm{NH}), 4.54-4.24\left(\mathrm{~m}, 5 \mathrm{H} ; \mathrm{H}-2, \mathrm{H}-6\right.$ and $\left.\mathrm{CH}_{2}\right), 4.17\left(\mathrm{t}, \mathrm{J}=6.8 \mathrm{~Hz}, 1 \mathrm{H} ; \mathrm{H}-9^{\prime}\right), 2.02-1.75(\mathrm{~m}, 4 \mathrm{H} ; \mathrm{H}-$ 3, H-5), $1.51-1.34$ (m, $2 \mathrm{H} ; \mathrm{H}-4)$ ppm. 
${ }^{13} \mathrm{C}$ NMR $\left(\mathrm{CDCl}_{3}, 150.9 \mathrm{MHz}\right): \delta=156.1(\mathrm{C}=0), 147.7$ (triazole-4), $143.6(\mathrm{Ar}), 141.3(\mathrm{Ar}), 130.0$ (triazole-5), 128.9, 128.4, 127.7, 127.1, 125.8, 125.1, 120.0, 119.8 (each $\mathrm{Ar}$ ), $67.1\left(\mathrm{OCH}_{2}\right), 53.4(\mathrm{C}-2)$, 50.0 (C-6), 47.1 (C-9'), 31.5 (C-3), 29.5 (C-5), 21.9 (C-4) ppm.

MS (ESI, MeOH): m/z calcd. $495.2[M-H]^{-}$; found 495.3.

\section{1,13-Bis(4-phenyl-1H-1,2,3-triazol-1-yl)-4,7,10-trioxatridecane (12)}<smiles>CCCOCCOCCOCCCn1cc(-c2ccccc2)nn1</smiles>

4,7,10-Trioxa-1,13-tridecanediamine $7(66 \mathrm{mg}, 0.30 \mathrm{mmol})$ and phenylacetylene $2(73 \mu \mathrm{l}, 0.66 \mathrm{mmol})$ were reacted according to general procedure 1. After flash chromatography (ethyl acetate / $\mathrm{MeOH}$ 20:1) 12 (141 mg, $99 \%$ ) was isolated as a white powder.

${ }^{1} \mathrm{H}$ NMR $\left(\mathrm{CDCl}_{3}, 600 \mathrm{MHz}\right): \delta=7.84(\mathrm{~s}, 2 \mathrm{H}$; triazole-5), $7.83-7.81(\mathrm{~m}, 4 \mathrm{H} ; \mathrm{Ar}-\mathrm{H}), 7.42-7.38(\mathrm{~m}, 4$ $\mathrm{H}$; Ar-H), $7.32-7.29(\mathrm{~m}, 2 \mathrm{H}$; Ar-H), $4.48(\mathrm{t}, \mathrm{J}=6.8 \mathrm{~Hz}, 4 \mathrm{H}$; H-1), $3.67-3.64(\mathrm{~m}, 4 \mathrm{H}, \mathrm{H}-5 \mathrm{or} \mathrm{H}-6)$, $3.59-3.57(\mathrm{~m}, 4 \mathrm{H}, \mathrm{H}-6$ or H-5), $3.42(\mathrm{t}, \mathrm{J}=5.8 \mathrm{~Hz}, 4 \mathrm{H} ; \mathrm{H}-3), 2.17-2.12(\mathrm{~m}, 4 \mathrm{H} ; \mathrm{H}-2)$ ppm.

${ }^{13} \mathrm{C}$ NMR $\left(\mathrm{CDCl}_{3}, 150.9 \mathrm{MHz}\right): \delta=147.5$ (triazole-4), 130.7, 128.7, 128.0, 125.6 (each $\mathrm{Ar}$ ), 120.4 (triazole-5), 70.5 (C-4 or C-5), 70.3 (C-5 or C-4), 67.0 (C-3), 47.1 (C-1), 30.1 (C-2) ppm.

MS (ESI, MeOH): $\mathrm{m} / z$ calcd. $499.2[\mathrm{M}+\mathrm{Na}]^{+} ;$found 499.4 .

Elemental Analysis: calcd. C $65.53 \%$, H $6.77 \%$, N $17.63 \%$; found: C $65.32 \%$, H $6.95 \%$, N $17.60 \%$.

\section{$\alpha, \alpha^{\prime}$-Bis(4-phenyl-1H-1,2,3-triazol-1-yl)-m-xylylene (13)}

$\alpha, \alpha^{\prime}$-Diamino- $m$-xylylene 8 (42 mg, $\left.0.31 \mathrm{mmol}\right)$ and phenylacetylene $2(75 \mu \mathrm{l}, 0.68 \mathrm{mmol})$ were reacted according to general procedure 1. After flash chromatography (petroleum ether / ethyl acetate 2:1 to ethyl acetate / MeOH 20:1) 13 (106 mg, $88 \%$ ) was isolated as a white powder.

${ }^{1} \mathrm{H}$ NMR (DMSO, $600 \mathrm{MHz}$ ): $\delta=8.62(\mathrm{~s}, 2 \mathrm{H}$; triazole-5), $7.84-7.82(\mathrm{~m}, 4 \mathrm{H}, \mathrm{Ar}-\mathrm{H}), 7.44-7.37(\mathrm{~m}, 6$ $\mathrm{H} ; \mathrm{Ar}-\mathrm{H}), 7.34-7.31$ (m, $4 \mathrm{H} ; \mathrm{Ar}-\mathrm{H}), 5.66\left(\mathrm{~s}, 4 \mathrm{H} ; \mathrm{CH}_{2}\right) \mathrm{ppm}$.

${ }^{13}$ C NMR (DMSO, $\left.150.9 \mathrm{MHz}\right): \delta=146.6$ (triazole-4), 136.6, 130.6, 129.3, 128.8, 127.9, 127.7, 127.4, 125.1 (each Ar), 121.5 (triazole-5), $52.8\left(\mathrm{CH}_{2}\right)$ ppm.

MS (ESI, MeOH): $\mathrm{m} / z$ calcd. $415.2[\mathrm{M}+\mathrm{Na}]^{+}$; found 415.1 .

Elemental Analysis: calcd. C $73.45 \%$, H $5.14 \%$, N $21.41 \%$; found: C $73.44 \%$, H $5.08 \%$, N $21.61 \%$. 


\section{Propargyl 2,3,4,6-Tetra-O-acetyl- $\beta$-D-glucopyranoside (15)}

2,3,4,6-Tetra-O-acetyl- $\alpha$-D-glucopyranosyl-trichloracetimidate ${ }^{6}(200 \mathrm{mg}, 0.41 \mathrm{mmol}$ ) and propargyl alcohol were dissolved in $2 \mathrm{ml}$ abs. $\mathrm{CH}_{2} \mathrm{Cl}_{2}$. Boron trifluoride etherate $(14 \mu \mathrm{l}$ of a $0.80 \mathrm{~m}$ stock solution in abs. $\mathrm{CH}_{2} \mathrm{Cl}_{2}, 0.011 \mathrm{mmol}$ ) was added at $0{ }^{\circ} \mathrm{C}$ and the reaction was stirred for $45 \mathrm{~min}$ at room temperature. Then, sat. $\mathrm{NaHCO}_{3}$ solution was added at $0{ }^{\circ} \mathrm{C}$. The aqueous phase was extracted once with dichloromethane. The combined organic phases were washed with water, dried over $\mathrm{Na}_{2} \mathrm{SO}_{4}$ and the organic solvent was removed under reduced pressure. After flash chromatography (petroleum ether / ethyl acetate 2:1) 15 (90 mg, $57 \%$ ) was isolated as a white powder. The ${ }^{1} \mathrm{H}$ and ${ }^{13} \mathrm{C}$ NMR spectroscopic data were in agreement with those published. ${ }^{7}$

\section{1,4-Bis(3-(4-(2,3,4,6-tetra-O-acetyl- $\beta$-D-glucopyranosyloxymethyl-1H-1,2,3-triazol-1-yl)-propoxy)- butane (16)}

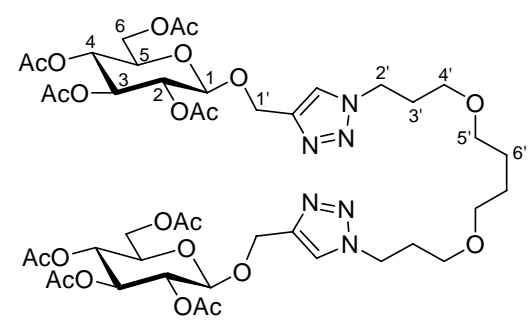

1,4-Bis(3-aminopropoxy)-butane (20 $\mathrm{mg}, 0.10 \mathrm{mmol})$ and $15(90 \mathrm{mg}, 0.23 \mathrm{mmol}$ ) were reacted according to general procedure 1 using 0.6 eq $\mathrm{NaN}_{3}$. After flash chromatography (dichloromethane I $\mathrm{MeOH}$ 19:1) 16 (89 mg, 86 \%) was isolated as a white powder.

${ }^{1} \mathrm{H} \mathrm{NMR}\left(\mathrm{CDCl}_{3}, 600 \mathrm{MHz}\right): \delta=7.51(\mathrm{~s}, 2 \mathrm{H}$; triazole-5), $5.16(\mathrm{t}, 2 \mathrm{H}, \mathrm{J}=9.5 \mathrm{~Hz} ; \mathrm{H}-3), 5.07(\mathrm{t}, 2 \mathrm{H}, \mathrm{J}=$ $9.6 \mathrm{~Hz} ; \mathrm{H}-4), 4.99$ (dd, $2 \mathrm{H}, \mathrm{J}=8.0$ and $9.6 \mathrm{~Hz} ; \mathrm{H}-2$ ), 4.91 (d, $2 \mathrm{H}, \mathrm{J}=12.6 \mathrm{~Hz} ; \mathrm{H}-1 \mathrm{a}$ ) $), 4.79$ (d, $2 \mathrm{H}, \mathrm{J}=$ $\left.12.5 \mathrm{~Hz} ; \mathrm{H}-1 b^{\prime}\right), 4.67$ (d, $2 \mathrm{H}, \mathrm{J}=8.0 \mathrm{~Hz}, \mathrm{H}-1$ ), 4.46-4.43 (m, $\left.4 \mathrm{H} ; \mathrm{H}-2^{\prime}\right), 4.25$ (dd, $2 \mathrm{H}, \mathrm{J}=4.8$ and 12.3 $\mathrm{Hz} ; \mathrm{H}-6 \mathrm{a}$ ), 4.13 (dd, $2 \mathrm{H}, \mathrm{J}=2.3$ and $12.3 \mathrm{~Hz} ; \mathrm{H}-6 \mathrm{~b}$ ), 3.72 (ddd, $2 \mathrm{H}, \mathrm{J}=2.4,4.7$ and $10.0 \mathrm{~Hz}$ ), $3.43-$ 3.38 (m, $8 \mathrm{H}$; H-4' and H-5'), 2.13 (quin., $4 \mathrm{H} ; \mathrm{H}^{\prime} 3^{\prime}$ ), 2.07 (s, $\left.6 \mathrm{H} ; \mathrm{C}(\mathrm{O}) \mathrm{CH}_{3}\right), 2.00\left(\mathrm{~s}, 6 \mathrm{H} ; \mathrm{C}(\mathrm{O}) \mathrm{CH}_{3}\right)$, 1.97 (s, $\left.6 \mathrm{H} ; \mathrm{C}(\mathrm{O}) \mathrm{CH}_{3}\right), 1.96$ (s, $\left.6 \mathrm{H} ; \mathrm{C}(\mathrm{O}) \mathrm{CH}_{3}\right), 1.64-1.61$ (m, $4 \mathrm{H} ; \mathrm{H}-6$ ') ppm.

${ }^{13} \mathrm{C}$ NMR $\left(\mathrm{CDCl}_{3}, 150.9 \mathrm{MHz}\right): \delta=170.6,170.1,169.4,169.3$ (each C=O), 143.9 (triazole-4), 122.9 (triazole-5), 99.8 (C-1), 72.7 (C-3), 71.9 (C-5), 71.2 (C-2), 70.8 (C-5'), 68.3 (C-4), 66.7 (C-4'), 62.9 (C1'), 61.8 (C-6), 47.4 (C-2'), 30.4 (C-3'), 26.4 (C-6'), 20.7, 20.6, 20.5 (each $\left.\mathrm{CH}_{3}\right)$ ppm.

MS (ESI, MeOH): $\mathrm{m} / z[M+\mathrm{Na}]^{+}$calcd. 1051.4; found 1051.4.

Elemental Analysis: calcd. C $51.36 \%$, H $6.27 \%$, N $8.17 \%$; found: C $51.13 \%$, H $6.26 \%$, N $8.22 \%$.

(1) Hassner, A.; Stern, M. Angew. Chem., Int. Ed. 1986, 25, 478-479. Hassner, A.; Stern, M.; Gottlieb, H. E. J. Org. Chem. 1990, 55, 2304-2306. Dharanipragada, R.; VanHulle, K.; Bannister, A.; Bear, S.; Kennedy, L.; Hruby, V. J. Tetrahedron 1992, 48, 4733-4748. Bretherick, L. Chem. Eng. News 1986, 64 
(Dec. 22), 2. Peet, N. P.; Weintraub, P. M. Chem. Eng. News 1993, 71 (Apr. 19), 4. Hruby, V. J.; Boteju, L.; Li, G. Chem. Eng. News 1993, 71 (Oct. 11), 2.

(2) Nyffeler, P. T.; Liang, C.-H.; Koeller, K. M.; Wong, C.-H. J. Am. Chem. Soc. 2002, 124, 1077310778. Lundquist, J. T.; Pelletier, J. C. Org. Lett. 2001, 3, 781-783.

(3) Raghavendra, M. S.; Lam, Y. Tetrahedron Lett. 2004, 45, 6129-6132.

(4) Abu-Orabi, S. T.; Atfah, M. A.; Jibril, I.; Mari'i, F. M.; Ali, A. A.-S. J. Heterocyclic Chem. 1989, 26, 1461-1468.

(5) Holzer, W. Tetrahedon 1991, 47, 9783-9792.

(6) Schmidt, R. R.; Michel, J. Angew. Chem. Int. Ed. Engl. 1980, 19, 731-732.

(7) Crombez-Robert, C.; Benazza, M.; Fréchou, C.; Demailly, G. Carbohydr. Res. 1998, 307, 355-359. 


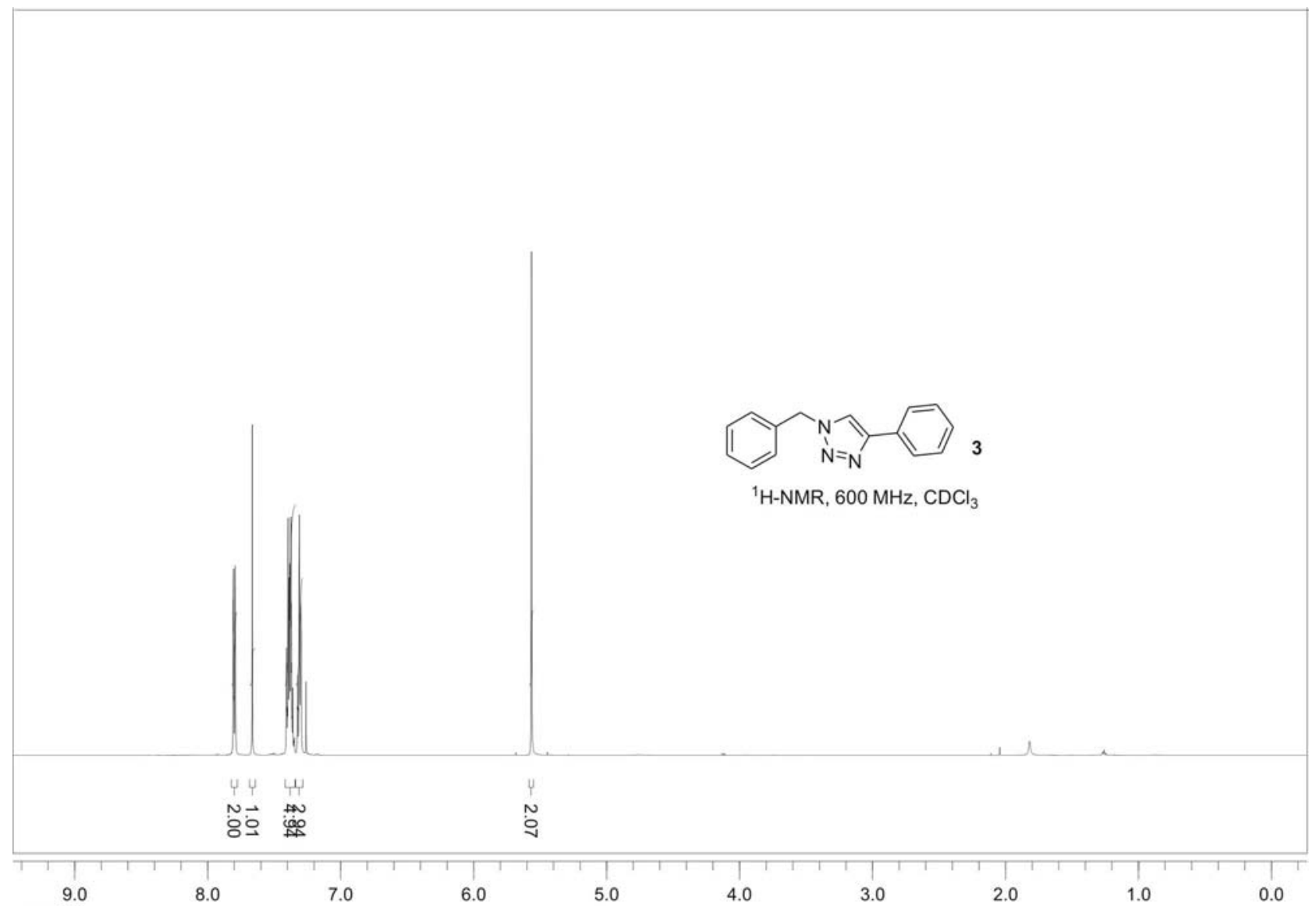

ppm (t1)
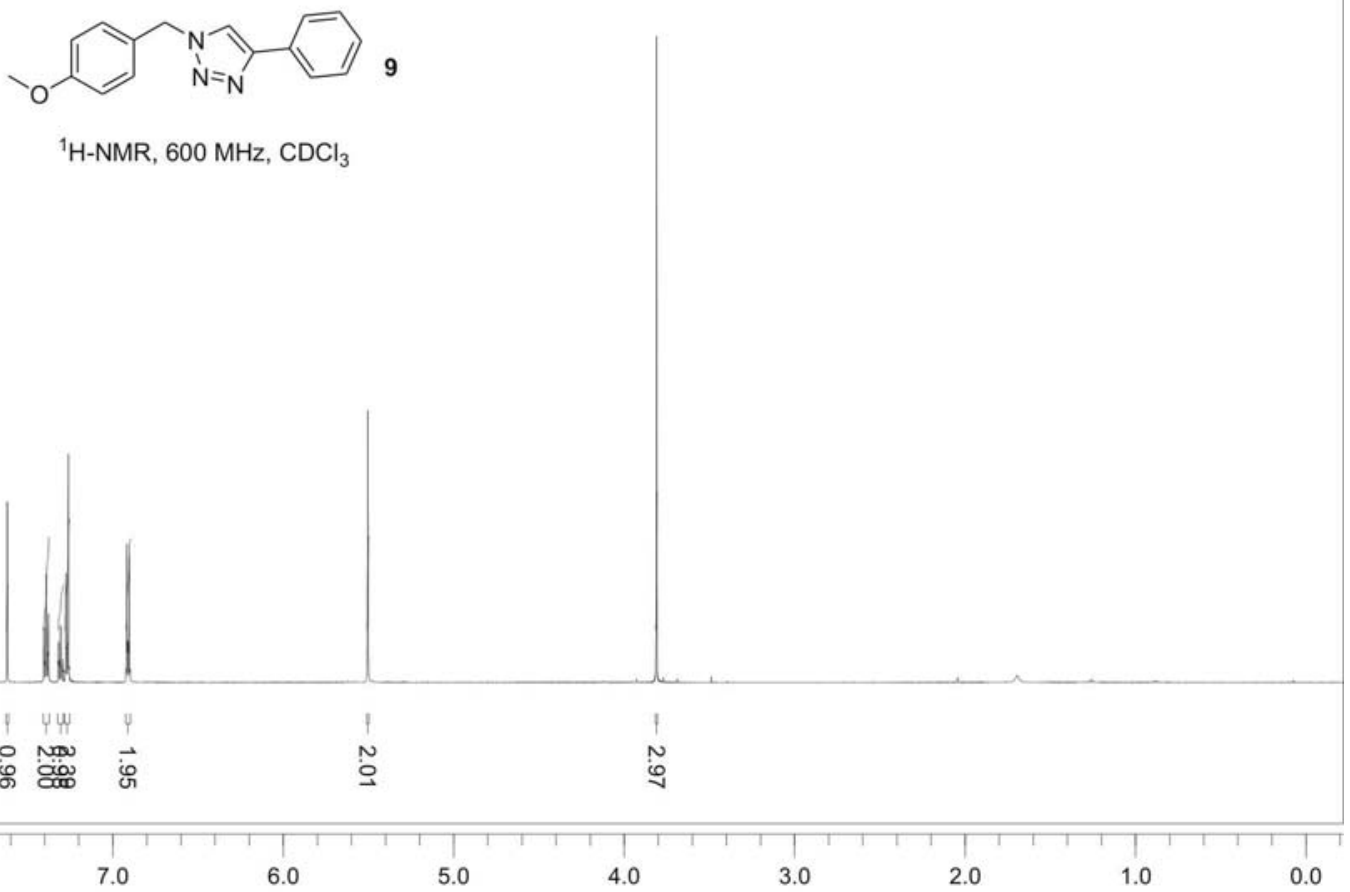

ppm (t1)

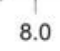

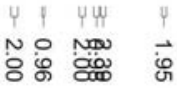

$\stackrel{1}{9}$

2.0 

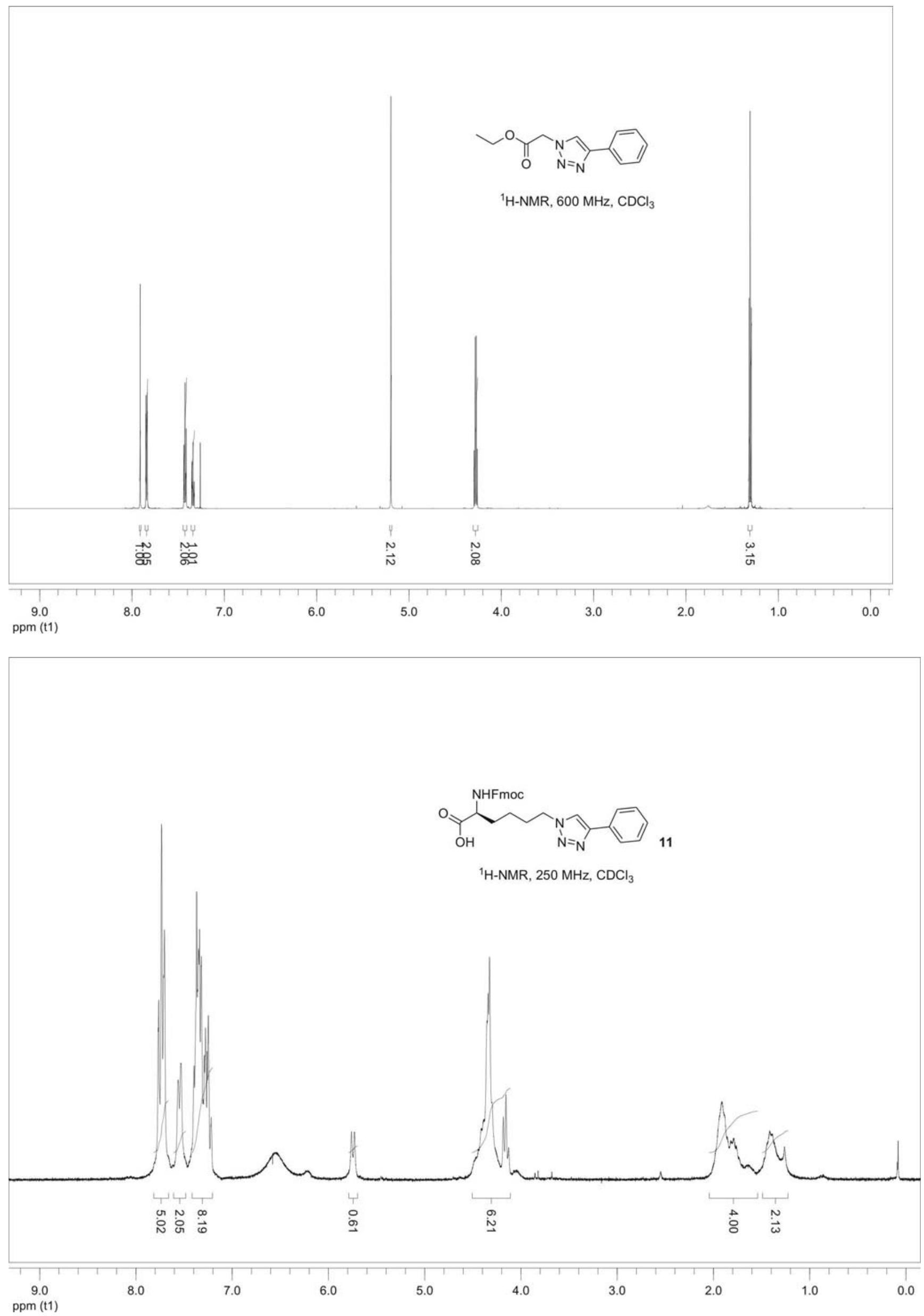

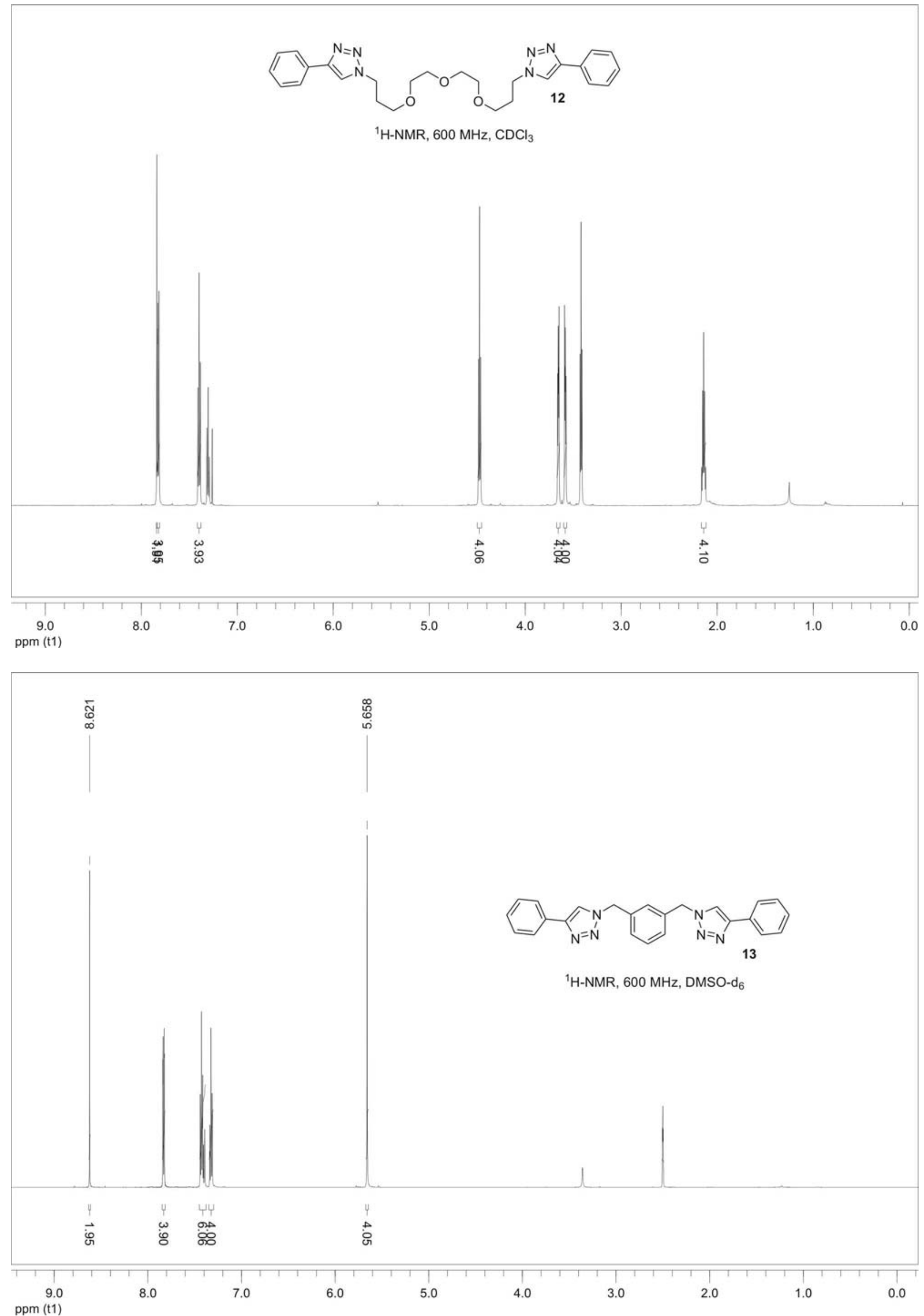

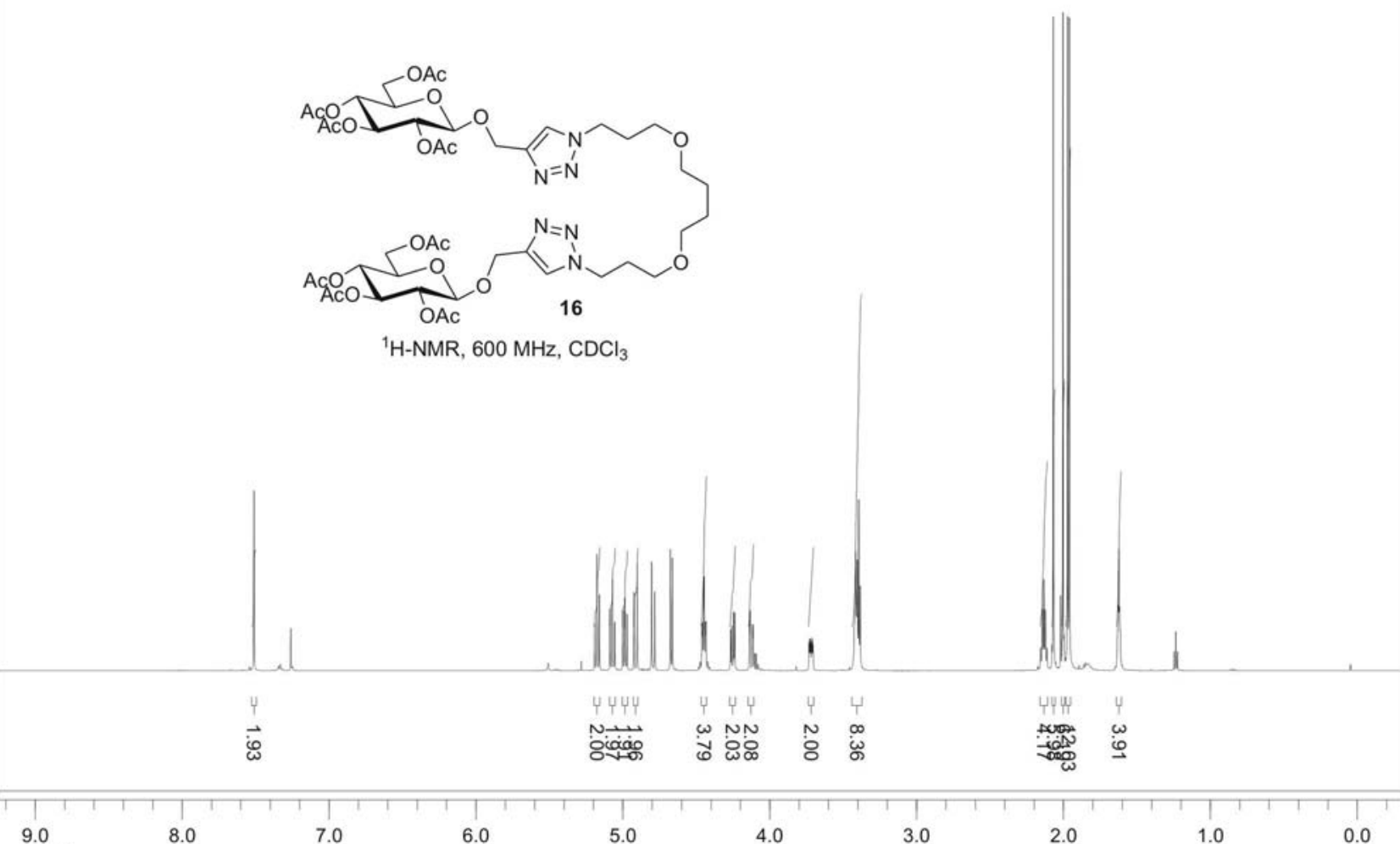

ppm (t1) 